\title{
The role of smoke from cooking indoors over an open flame and parental smoking on the risk of cleft lip and palate: A case- control study in 7 low-resource countries
}

\author{
Allyn Auslander ${ }^{1,3}$, Roberta \\ McKean-Cowdin ${ }^{1,2}$, Frederick \\ Brindopke $^{3,4}$, Beau Sylvester ${ }^{3}$, \\ Melissa DiBona ${ }^{4}$, Kathy Magee ${ }^{4}$, \\ Rijuta Kapoor ${ }^{1}$, David V Conti ${ }^{1}$, \\ Sylvia Rakotoarison ${ }^{5}$, William \\ Magee III ${ }^{1,3}$; Operation Smile \\ International Family Study \\ Group*
${ }^{1}$ Department of Preventive Medicine, Keck School of Medicine of the University of Southern California, California, USA
${ }^{2}$ USC Eye Institute, Department of Oph- thalmology, Keck School of Medicine of the University of Southern California, California, USA
${ }^{3}$ Children's Hospital Los Angeles, Los Angeles, California, USA
${ }^{4}$ Operation Smile, Inc.; Virginia Beach, Virginia, USA
${ }^{5}$ Operation Smile Madagascar; Antananarivo, Madagascar, USA
* See Acknowledgements for members/ affiliations of the Operation Smile Study Group

\section{Correspondence to:}

Allyn Auslander, MPH

USC Department of Preventive Medicine Keck School of Medicine of the University of Southern California

2001 N. Soto Street

Los Angeles, CA 90033

USA

aausland@usc.edu

\begin{abstract}
Background Cleft is one of the most common birth defects globally and the lack of access to surgery means millions are living untreated. Smoke exposure from cooking occurs infrequently in developed countries but represents a high-proportion of smoke exposure in less-developed regions. We aimed to study if smoke exposure from cooking is associated with an increased risk in cleft, while accounting for other smoke sources.
\end{abstract}

Methods We conducted a population-sampled case-control study of children with cleft lip and/or palate and healthy newborns from Vietnam, Philippines, Honduras, Nicaragua, Morocco, Congo, and Madagascar. Multivariable regression models were used to assess associations between maternal cooking during pregnancy, parental smoking, and household tobacco smoke with cleft.

Results 2137 cases and 2014 controls recruited between 2012-2017 were included. While maternal smoking was uncommon ( $<1 \%), 58.3 \%$ case and $36.1 \%$ control mothers cooked over an open fire inside. Children whose mothers reported cook smoke exposure were 49\% (95\% confidence interval $(\mathrm{CI})=1.2-1.8)$ more likely to have a child with a cleft. This was consistent in five of seven countries. No significant associations were found for any other smoke exposure.

Conclusions Our finding of maternal cook smoke and cleft in low-resource countries, similar to maternal tobacco smoke in high-resource countries, may reflect a common etiology. This relationship was present across geographically diverse countries with variable socioeconomic statuses and access to care. Exposures specific to low-resource settings must be considered to develop public health strategies that address the populations at increased risk of living with cleft and inform the mechanisms leading to cleft development.

Surgically treatable conditions account for approximately one-third of all global disease and an additional 2.2 million providers would be needed to treat the existing surgical need [1]. Although such conditions are treatable, many impacted children and adults do not have adequate access to care. Prevention of surgically treatable birth defects is therefore a necessary goal as provision of surgical treatment for all patients is unlikely - especially for diseases such as orofacial clefts that require complex multidisciplinary care.

Cleft lip with or without palate is one of the most common birth defects worldwide. The global incidence is approximately 1 in 700 live births [2], however incidence ranges from 1.28-1.90 per 1000 live births in Asians, 0.96-1.87 per 1000 in Hispanics, 1 per 1000 in non-Hispanic Whites, and 0.73-1.22 per 1000 
in populations of African descent [2-5]. Although there is clear variability by ethnicity, the majority of etiologic data comes from individuals of European descent [6]. Disruptions in craniofacial development occur during the first trimester of pregnancy during weeks 4 to 13 of development [7]. Cleft is characterized as an embryologic failure of fusion of facial elements that normally develop into the lip and palate. In the absence of any other birth defect, the patient is considered to be non-syndromic (approximately $70 \%$ of cleft lip with or without palate and $50 \%$ of isolated cleft palate patients) $[2,6-8]$. While the origins of syndromic clefts are considered largely genetic, the etiology of non-syndromic clefts remains unclear.

Parental smoking has been considered an important determinant of developmental disorders, but the environmental impact of smoke exposure from cooking and cleft risk has only been mentioned in two existing studies $[9,10]$. In low-resource countries, biomass cooking fuel is used by approximately $80 \%$ of rural households and has been associated with a wide variety of diseases, including stillbirths [11]. While maternal smoking has been associated with risk of cleft [12-14], the association between smoke from cooking remains uncharacterized. Beyond different types of smoke exposure, the main established risk factors for cleft are low maternal education [15,16], lack of folic acid supplementation [17,18], advanced maternal age [19,20], family history of clefts [21], and ethnicity [22]. Other factors that have been less consistently associated with cleft are periconceptional alcohol use [23,24] and diabetes (either pregestational or gestational) [25,26].

In the current analysis, we used data from over 4000 children and their mothers collected on surgical missions conducted by Operation Smile. Specifically, we assessed the relationship between smoke exposure from cooking and the risk of non-syndromic cleft. Other sources of smoke investigated include maternal smoking, paternal smoking, and household tobacco smoke. This study is the first to evaluate cooking practices as an environmental determinant of cleft in a population-recruited sample of children from diverse, low-resource countries. Data were collected from 7 countries (Vietnam, Philippines, Morocco, Madagascar, Democratic Republic of Congo (DRC), Honduras, Nicaragua) to evaluate the association overall and to explore factors that may influence heterogeneity of effects by country. Clarifying the role of prenatal exposure to smoke from sources common to different populations and cleft risk may help to improve our understanding of risk factors contributing to non-syndromic cleft and inform preventive strategies.

\section{METHODS}

Data for this study was collected from 2012-2017 as part of a coordinated series of population-sampled case-control studies focusing on genetic, lifestyle and environmental exposures and cleft in children 6 months to 4 years of age. This study was conducted with Operation Smile (OS), an internationally recognized not-for-profit that has been providing free cleft surgery and related care to patients for over 36 years. Data for the current analysis represents children from 7 countries sampled over multiple missions (Table S1 in the Online Supplementary Document). Participation rates in the study varied by site from 77\%-96\% for cases and 45\%-100\% for controls. The methods of this study have been previously published in depth $[25,27]$. All work was approved by the Institutional Review Board at the University of Southern California including country-specific authorizations.

\section{Case definition}

This study includes non-syndromic cases of cleft lip and / or cleft palate (ICD10 35-37) [28] Cleft lip and palate (CLP) is the most common phenotype, followed by isolated cleft lip (iCL) and isolated cleft palate $(\mathrm{iCP})$. Cleft lip with or without palate (CL+/-P) is used to denote CLP and iCL. Cases were screened to confirm diagnosis and absence of any genetic syndrome or other birth defect by medical practitioners at the mission site. This included pediatricians, nurses, anesthesiologists and surgeons who are all formally licensed, trained and OS certified to work with cleft patients.

Patients were included in the study if they were accompanied by their biological mother (18 years or older), 6 months to 4 years of age, and presented for cleft treatment at the time of the OS mission. Patients were excluded if the child was not the most recent pregnancy, a multiple birth, had a genetic syndrome, or had another co-morbid condition.

\section{Case recruitment}

Cases for the International Family Study (IFS) are recruited on site during OS missions. IFS countries were selected from sites OS identified a priori as having adequate infrastructure to support research and 
the specific hospital was chosen based on its ability to meet the organization's 'Global Standards of Care'. Extensive regional recruitment and community outreach efforts are conducted by OS prior to each mission to assure saturation of the communities. All patients arrive to the mission site to be screened for care over the span of one or two days with all costs covered by OS. The patients are registered and seen by general practitioners, nurses, anesthesiologists, surgeons, and dentists to assess surgical eligibility. Case recruitment for the study occurs at the end of the screening process. Study eligibility criteria were identical for cases in all countries.

\section{Control definition}

Controls were newborns identified from regional neighborhood, clinic, and hospital-based birth centers around the mission site (Table S1 in the Online Supplementary Document) whose mother agreed to complete informed consent and the study interview. Individuals were excluded if they had a cleft or any other birth defect, were a multiple birth, or if the mother was younger than 18 .

\section{Control recruitment}

Multiple neighborhood, clinic, and hospital-based birth centers were identified prior to each mission by in-country OS partners to represent the catchment area of the OS mission and improve case-control comparability. All maternity wards selected were public to better match demographics of the mission patients. The leadership at the birth center was approached and debriefed on the study, and local authorization was obtained to recruit families along with IRB approval prior to the mission. Each site was visited daily during the mission.

\section{Data collection}

Local volunteers with medical training (ie, nursing/medical students) were identified by OS and underwent training by study members. Local interviewers were used to assure high recruitment and allow completion of the interview in the language of the families; however, the study supervisor was present during all interviews for consistency and to maintain quality. Informed consent was completed before the interview and parents were assured that participation was not required for their child to receive care. Families were interviewed in a private to semi-private area (depending on screening space). Questionnaires have been translated and back translated by certified translators to ensure consistency across countries.

Mother's interviews took approximately 40 minutes. The interview included questions on family history of cleft, lifestyle and environmental exposures (smoke, alcohol, diet, water source), medical history (parental medical history, use of prescription and nonprescription drugs), demographics (age, pregnancy history, education, employment), and paternal factors (smoke, alcohol, age, employment, education). When the father was present, a limited interview is independently completed on medical history, environmental, and lifestyle exposures. Our current analysis included data exclusively from the mothers' interviews.

\section{Statistical analysis}

Descriptive statistics, including proportions for categorical variables and means for continuous variables, were constructed for the child characteristics, parental characteristics, and lifestyle factors. Tests of statistical significance included $t$ tests for continuous and $\chi^{2}$ tests for categorical variables. Maternal exposure to indoor cook smoke was categorized as a dichotomous variable (yes/no). Maternal smoking was dichotomized (ever/never) for the three months prior to pregnancy and during pregnancy. Smoking pre-pregnancy was not collected in Vietnam, so they are not included in those analyses. Fathers were dichotomized into lifetime smokers (ever) or never smokers and the definition included any type of tobacco product (cigarettes, pipes, chewing tobacco, cigars, other). Household tobacco smoke is defined as any member of the household smoking inside during the mother's pregnancy with the child (yes/no). All education variables were harmonized as less than secondary school or secondary school or higher for mothers and fathers separately. Family history of cleft was defined as any first or second degree relative having any cleft. Number of children was classified into 1, 2, or 3 or more.

Logistic regression was used to calculate odds ratios and 95\% confidence intervals for smoke exposure (indoor cook smoke, maternal smoking, paternal or household tobacco smoke) and cleft overall and by subtype (iCP, CL+/-P). Models were specified as minimal (adjusted for country, maternal age at the child's birth, mother's education, father's education, and family history of cleft), full (adjusted for minimal model and rural/urban residence and maternal alcohol consumption during pregnancy), and a mutual- 


\section{RESULTS}

A total of 4426 eligible children were identified from the 7 countries between 2012 and 2017. Of these: 58 participants were excluded due to missing case status and an additional 217 were excluded because they exceeded the newborn to four-year inclusion criteria. 4151 participants were included in the final data set: 2137 cases (51\%) and 2014 controls (49\%) with the majority coming from Vietnam (31.8\%), followed by the Philippines (22.3\%), Honduras (22.1\%), Congo (10.2\%), Madagascar (5.1\%), Morocco (4.3\%), and Nicaragua (4.2\%). Cases and controls were recruited simultaneously in all years the study was active with the exception of a delay in control collection in Vietnam in 2012 due to approval delays (Table S2 in the Online Supplementary Document). The case phenotype distribution consisted of 1198 (56.1\%) with CLP, 553 (25.9\%) with iCL, and 306 (14.3\%) with iCP (Table 1).

Characteristics of the study population are described in Table 1, Table 2 and Table 3. Case mothers were on average six months older than controls $(P=0.008)$ and less often employed $(P=0.03)$. Control mothers $(81.1 \%$ 


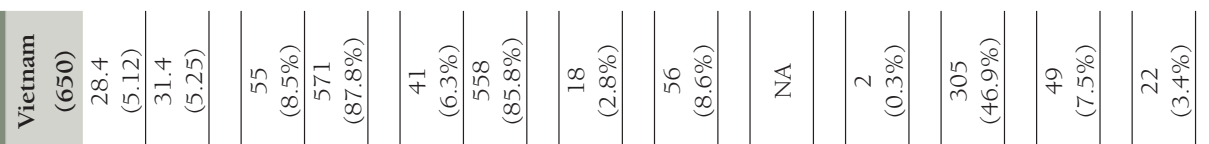

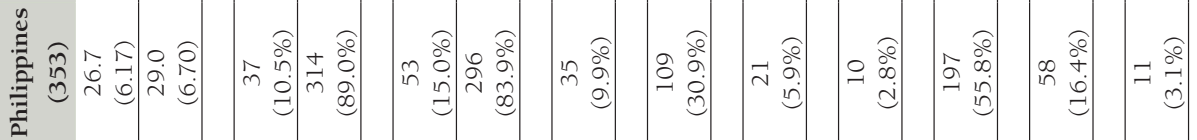

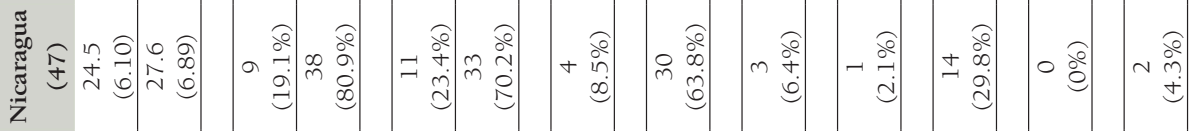

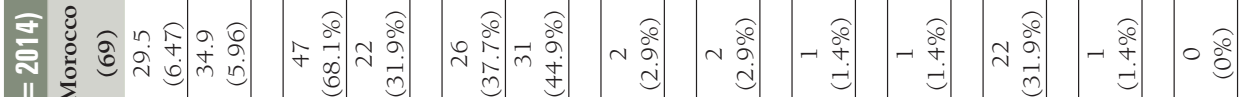

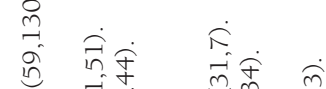

言咅

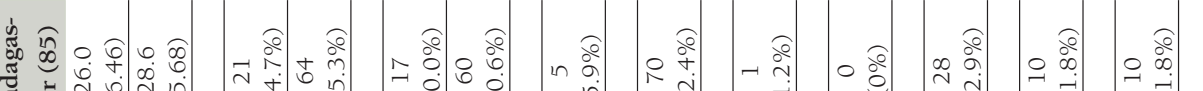

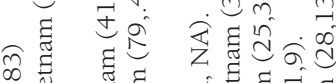

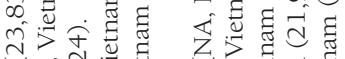

घ $0.5 \%$

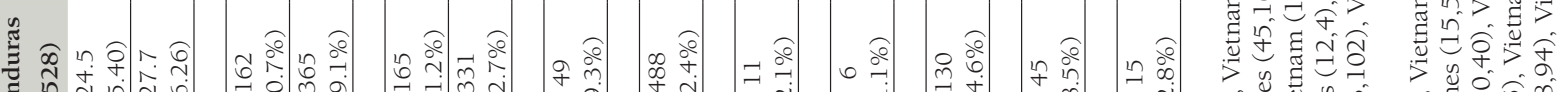

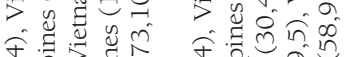

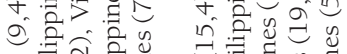

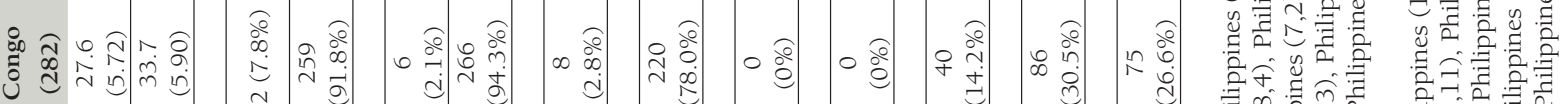

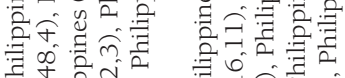

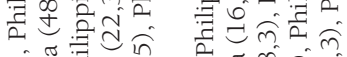

z

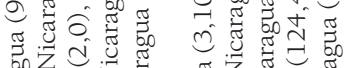

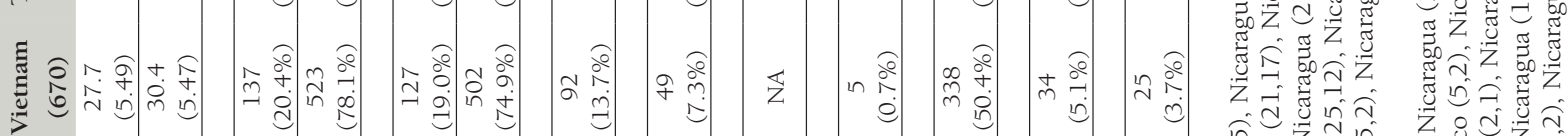

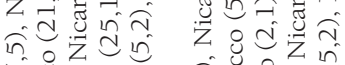

5.

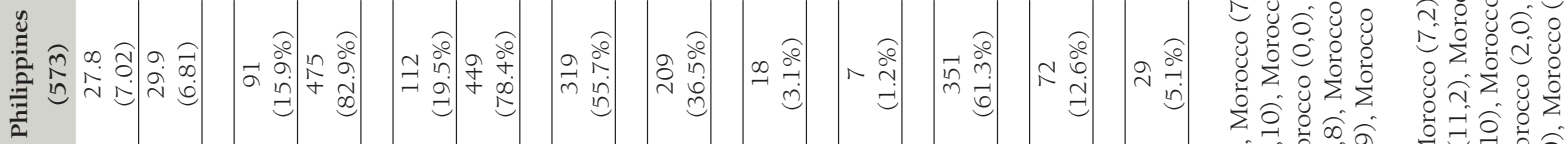

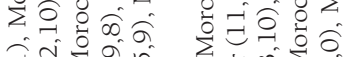

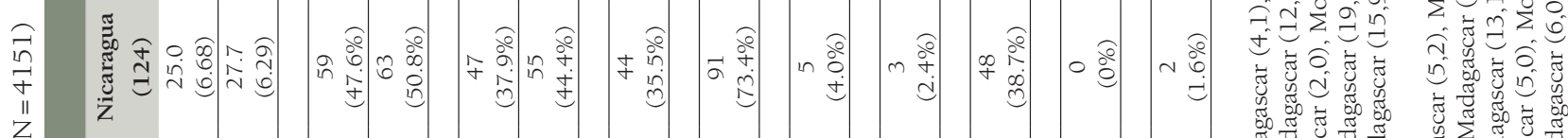

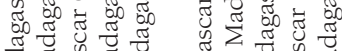

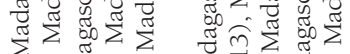

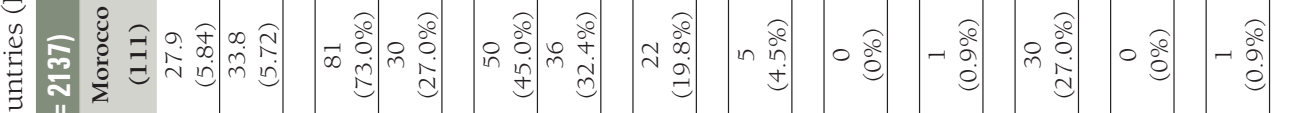

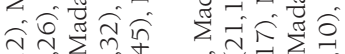

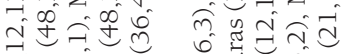

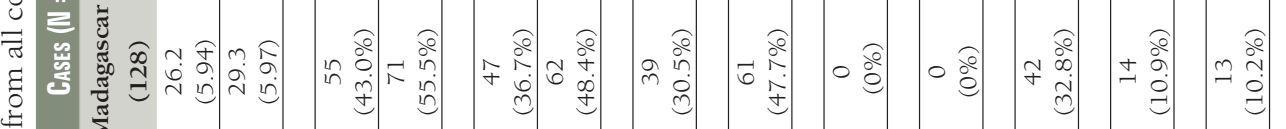

要造

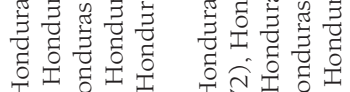

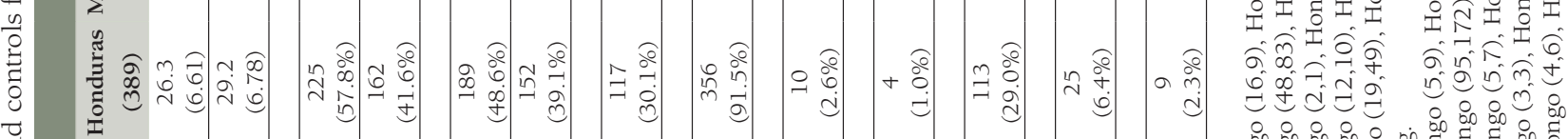

च

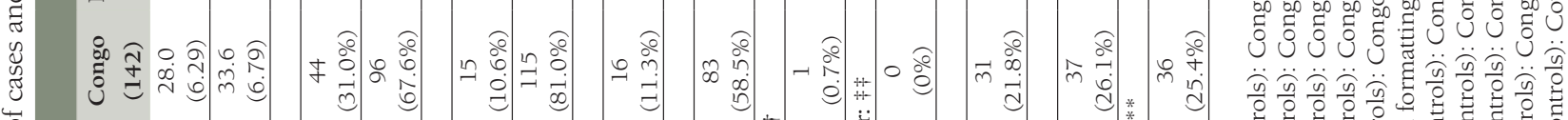

प्र 0 (a)

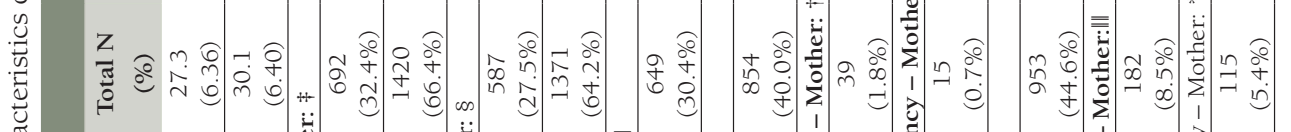

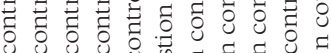
$F=A$ F

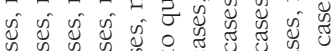

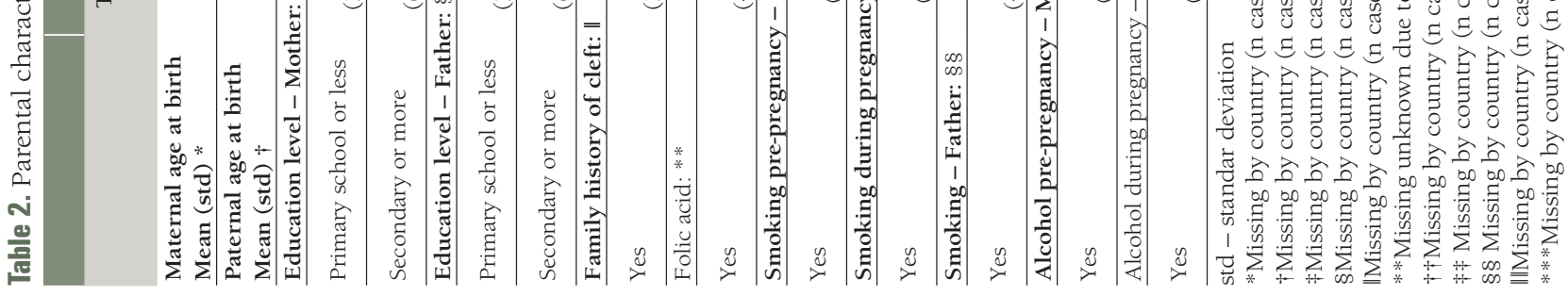

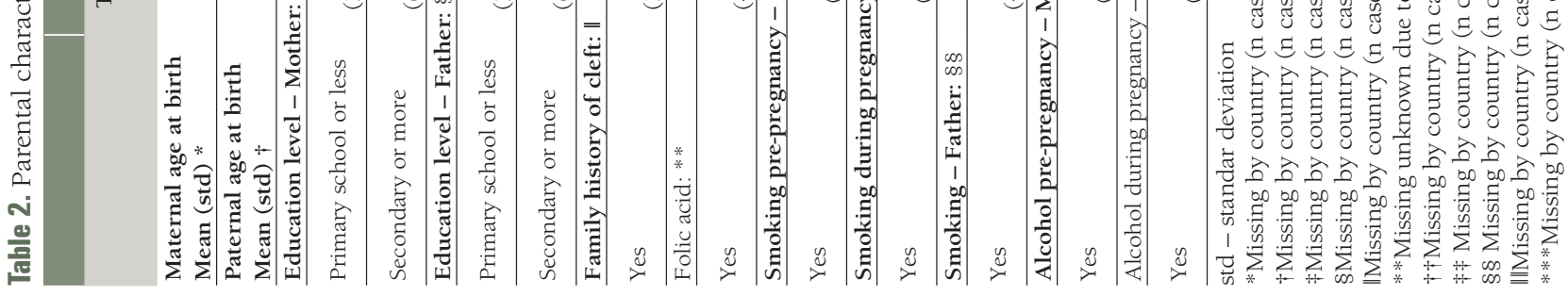




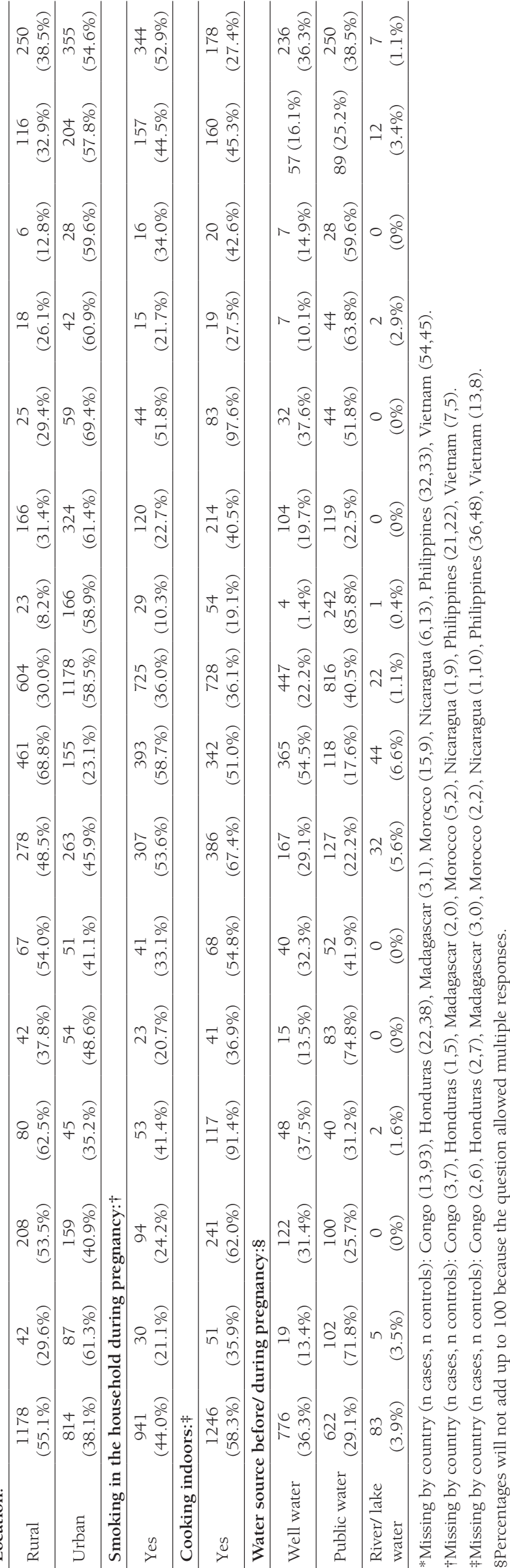

vs $66.4 \%)$ and fathers ( $78.2 \%$ vs $64.2 \%$; both $P<0.001)$ were more likely to have a secondary education. No difference was observed in father's age, father's employment status, or maternal smoking (prior to or during pregnancy). A higher proportion of cases reported cooking indoors over an open flame (58.3\% vs $36.1 \%)$. Fewer case mothers reported drinking alcohol pre-pregnancy $(8.5 \%$ vs $12.4 \% ; P<0.001)$; however, they were more likely to report drinking during pregnancy $(P=0.09)$, living in a rural area, smoking in the household, and that the father of the child smoked (all $P<0.001$ ). The distribution of all five smoking variables significantly differed across countries (all $P<0.05$ ).

The relationship between smoke exposures and the odds of all cleft is shown in Table 4. A strong positive association was found between cooking indoors over an open flame and risk of all cleft types. Mothers who reported cooking over an open flame indoors were $49 \%$ more likely to have a child with a cleft after adjusting for country, maternal age, mother and father education, family history of cleft, rural/ urban residence, alcohol consumption during pregnancy and all smoke variables [Model 3]. With respect to maternal smoking during pregnancy, the odds ratio (OR) was elevated but the confidence interval included the null $(\mathrm{OR}=1.65,95 \%$ confidence interval $(\mathrm{CI})=0.5,5.6)$. The prevalence of mothers who smoked prior to or during pregnancy was low (prior: 39 cases (1.8\%) and 39 controls (1.9\%); during: 15 cases (0.7\%) and 18 controls $(0.9 \%))$. No association was found with smoking the three months prior to pregnancy or exposure to household tobacco smoke. There was a positive association with ever paternal smoking and cleft $(\mathrm{OR}=1.18,95 \% \mathrm{CI}=0.96,1.5)$ however the confidence interval included the null. The results of Table 4 were nearly identical when restricted to women with no history of smoking $(n=4057)$. No evidence of interaction by country was found (all $P>0.05$ ).

The analyses restricting to $\mathrm{CL}+/-\mathrm{P}$ (cases $=1751$; excluding iCP) are explored in Table 5. Cooking indoors over an open flame was associated with a modest increase in risk of cleft $(\mathrm{OR}=1.55$, $95 \% \mathrm{CI}=1.3,1.9)$ compared to the full case set. For iCP (cases $=306$ ), only cooking indoors over an open flame in the minimally adjusted model showed elevated risk $(\mathrm{OR}=1.65,95 \% \mathrm{CI}=1.2$, 2.3) (Table $S 2$ in the Online Supplementary Document). No other smoking variables were associated with iCP in any model.

The impact of rural/ urban residence, parental education, and case age on risk of all cleft types was explored in stratified analyses. Cooking indoors over an open flame was associated with 
Table 4. Adjusted odds ratios (OR) of smoke related factors and cleft lip and/or palate in all countries $(\mathrm{N}=4151)$

\begin{tabular}{|c|c|c|c|c|c|c|c|c|c|}
\hline & \multicolumn{3}{|c|}{ ModeL $1^{*}$} & \multicolumn{3}{|c|}{ MODEL 2 † } & \multicolumn{3}{|c|}{ MODEL 3 † } \\
\hline & OR & $95 \%$ CI & $P$-value & OR & $95 \%$ CI & $P$-value & OR & $95 \%$ CI & $P$-value \\
\hline Cooking indoors over open flame & 1.93 & $(1.64,2.27)$ & $<.0001$ & 1.51 & $(1.26,1.81)$ & $<.0001$ & 1.49 & $(1.23,1.79)$ & $<.0001$ \\
\hline Smoking Pre-pregnancy- Mother & 0.88 & $(0.49,1.58)$ & 0.67 & 0.92 & $(0.49,1.70)$ & 0.78 & 1.65 & $(0.50,5.61)$ & 0.52 \\
\hline Smoking during pregnancy- Mother & 1.20 & $(0.45,3.34)$ & 0.72 & 1.39 & $(0.49,4.05)$ & 0.54 & 0.79 & $(0.38,1.61)$ & 0.41 \\
\hline Smoking- Father & 1.11 & $(0.95,1.30)$ & 0.19 & 1.10 & $(0.93,1.31)$ & 0.28 & 1.18 & $(0.96,1.47)$ & 0.12 \\
\hline Smoking in the household during pregnancy & 1.04 & $(0.89,1.23)$ & 0.61 & 0.98 & $(0.83,1.17)$ & 0.86 & 0.85 & $(0.68,1.06)$ & 0.14 \\
\hline
\end{tabular}

OR - odds ratio, $\mathrm{CI}$ - confidence interval

*Model 1 - Adjusted for country, maternal age, mother and father education (primary or less/ secondary or more), family history of cleft.

$\uparrow$ Model 2 - Additionally adjusted for rural/ urban home and alcohol consumption during pregnancy.

\$Model 3 - Mutually adjusted for all smoke variables and Model 2 covariates.

Table 5. Adjusted odds ratios (OR) of smoke related factors and cleft lip with or without cleft palate (excluding iCP) in all countries $(\mathrm{N}=3765)$

\begin{tabular}{|c|c|c|c|c|c|c|c|c|c|}
\hline & \multicolumn{3}{|c|}{ Model $1^{*}$} & \multicolumn{3}{|c|}{ MODEL 2 † } & \multicolumn{3}{|c|}{ MODEL 3 ‡ } \\
\hline & OR & $95 \%$ CI & $P$-value & OR & $95 \%$ CI & $P$-value & OR & $95 \%$ CI & $P$-value \\
\hline Cooking indoors over open flame & 2.02 & $(1.70,2.41)$ & $<.0001$ & 1.56 & $(1.29,1.90)$ & $<.0001$ & 1.55 & $(1.27,1.89)$ & $<.0001$ \\
\hline Smoking Pre-pregnancy- Mother & 0.91 & $(0.50,1.66)$ & 0.76 & 0.95 & $(0.50,1.80)$ & 0.87 & 2.08 & $(0.61,7.30)$ & 0.43 \\
\hline Smoking during pregnancy- Mother & 1.43 & $(0.53,4.02)$ & 0.48 & 1.65 & $(0.57,4.86)$ & 0.35 & 0.74 & $(0.34,1.57)$ & 0.24 \\
\hline Smoking- Father & 1.08 & $(0.92,1.28)$ & 0.35 & 1.08 & $(0.90,1.29)$ & 0.42 & 1.14 & $(0.91,1.43)$ & 0.26 \\
\hline Smoking in the household during pregnancy & 1.06 & $(0.89,1.25)$ & 0.54 & 1.00 & $(0.83,1.20)$ & 0.98 & 0.87 & $(0.69,1.10)$ & 0.25 \\
\hline
\end{tabular}

$\mathrm{CI}$ - confidence interval, $\mathrm{OR}$ - odds ratio, iCP - cleft palate only

*Model 1 - Adjusted for country, maternal age, mother and father education (primary or less/ secondary or more), family history of cleft.

$\uparrow$ Model 2 - Additionally adjusted for rural/ urban home and alcohol consumption during pregnancy.

¥Model 3 - Mutually adjusted for all smoke variables and Model 2 covariates.

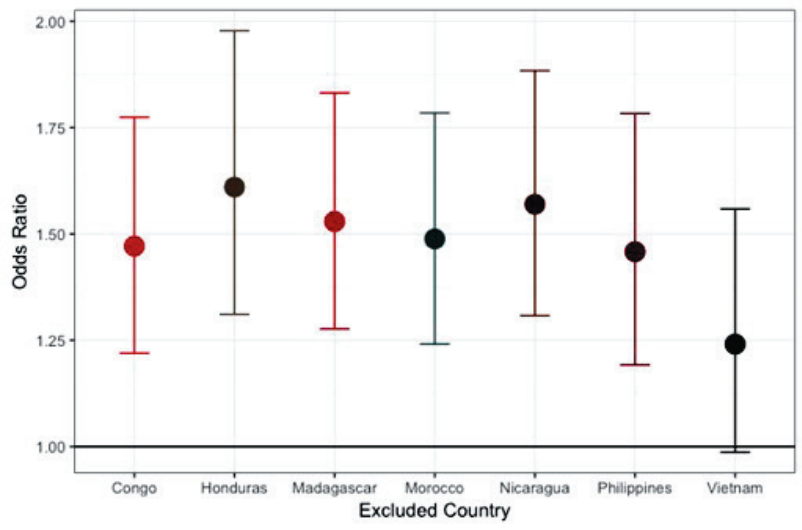

Figure 1. Cooking indoors over an open flame - odds ratio and 95\% confidence interval excluding each country (all cleft types combined).

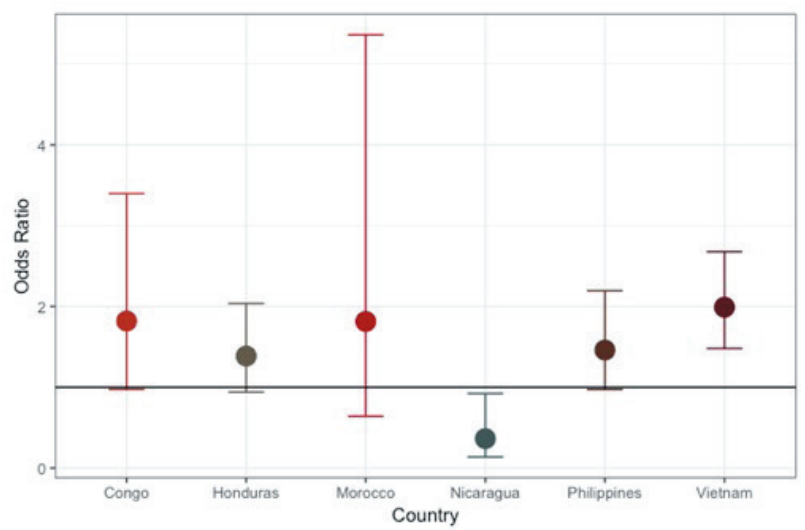

Figure 2. Cooking indoors over an open flame - odds ratio and 95\% confidence interval by country (all cleft types combined). cleft in both rural and urban residence. However, the magnitude was higher for urban $(\mathrm{OR}=1.71,95 \% \mathrm{CI}=1.3,2.3)$ than rural $(\mathrm{OR}=1.28,95 \% \mathrm{CI}=1.0,1.7)$ (data not shown). Sensitivity analyses showed that the results were as strong by education group for both parents (less than secondary vs secondary or higher) using stratification, by paternal smoking status (yes v. no) using stratification, after adjusting for income, and when restricting to cases under one year of age (group most comparable to newborn controls).

Effect estimates after exclusion of individual countries are shown in Figure 1. The overall effect was not dramatically influenced by data from any single country with the exception of Vietnam. The association was slightly reduced $(\mathrm{OR}=1.25(1.0,1.6))$ with the removal of Vietnam, which contributed $31.8 \%$ of the data set. The individual association of cooking indoors over an open flame by country is shown in Figure 2. Positive associations were found in every country besides Nicaragua. The most extreme results were observed in Vietnam (OR=2.05, 95\% CI=1.5, 2.8) followed by the Congo $(\mathrm{OR}=1.82,95 \% \mathrm{CI}=1.0,3.4)$, and the Philippines $(\mathrm{OR}=1.47,95 \% \mathrm{CI}=1.0,2.2)$. Madagascar did not contribute to the figures due to minimal variation $(93.9 \%$ reported cooking indoors over an open flame). The results observed for CL+/-P were comparable and slightly further from the null with Congo, Honduras and the Philippines reaching statistical significance (Figure S1 and S2 in the Online Supplementary Document). The iCP results showed a generally positive association with cooking indoors over an open flame (Figure S3 and S4 in the Online Supplementary Document). 


\section{DISCUSSION}

This is the first study to report on the association between cooking indoors over an open flame and non-syndromic cleft using data from multiple low-resource countries. Mothers who reported in-door cook smoke exposure were approximately 50\% more likely to have a child with a cleft. This result was present after controlling for suspected confounders (including other sources of smoke exposure) and in countries with different socioeconomic characteristics and access to care, as well as being consistent for 5 of 7 countries (Vietnam, the Philippines, Honduras, DRC, and Morocco). The negative association found in Nicaragua was underpowered and will need to be explored further as the sample size increases. No association was found with maternal smoking (prior to or during pregnancy), paternal smoking (lifetime), environmental tobacco smoke, or folic acid supplementation.

Similar to our findings, a prior case-control study by Liu et al. in China found that indoor air pollution was associated with an increased odds of cleft if the house was not ventilated (OR=4.5, 95\% CI = 1.612.9)) and attributed this to coal-burning heating sources [9]. A second study in the Congo found the odds of cleft was 6-times higher among mothers who reported cooking indoors [10]. However, the sample size was small ( $\mathrm{n}=162$ cases; 162 matched controls) and they didn't adjust for additional confounding factors beyond matching. Notably, they also found no maternal smoking effect but did find a positive association with paternal smoking.

Although we did not observe an effect of mother's smoking on the risk of cleft, the frequency of maternal smoking was very low, and smoking is most often not economically accessible or culturally accepted for women in low-resource populations. According to 2016 World Bank data, 1\% of women smoke in Vietnam, $7.8 \%$ in the Philippines, $2 \%$ in Honduras, and $0.80 \%$ in Morocco [29]. Although the US Surgeon General report identified maternal smoking during pregnancy as a risk factor for cleft [30], it is unclear if these findings are generalizable to low-resource settings. The literature consistently supports an association between maternal smoking and risk of cleft. Specifically, a meta-analysis of 23 case-control and 6 cohort studies found that mothers who smoked were 37\% more likely to have a child with CL+/-P than never smokers [12,14,31]. It is worth noting that 27 of 29 studies were conducted in populations of European decent and developed countries, which may reflect a different risk profile than the individuals in our study.

The effects of paternal smoking and ETS on cleft is less conclusive. Paternal smoking was evaluated in a subset of the current data from Vietnam, the Philippines, Morocco, and Honduras ( $\mathrm{n}=626$ father/child duos) and no association with cleft was found [32]. Studies in Norway [33], India [34], and China [35] have found that exposure to ETS, defined as an exposure to passive tobacco smoke during the first trimester at home or work, is associated with an increased risk of cleft ( $\mathrm{OR}=1.6,95 \% \mathrm{CI}=1.0-2.5 ; \mathrm{OR}=2.0$, $95 \% \mathrm{CI}=1.2-3.4 ; \mathrm{OR}=2.46,95 \% \mathrm{CI}=0.99-6.08$, respectively), however a case-control study based in a large American birth defect registry found no effect [31]. Our study did not see an effect of either paternal smoking or ETS, where ETS is defined as maternal report of passive smoke exposure in the home during pregnancy.

The mechanism for a role of smoke on cleft formation in embryonic development has been described in the current literature. An association between maternal periconceptional exposure to secondhand tobacco smoke and cleft in offspring has been consistently found [36-41] in epidemiological studies, while 2 studies have linked clefting with maternal exposure to other indoor air pollutants and combustion byproducts [9,42]. The incomplete combustion of tobacco and other organic compounds, including fuels for cooking and heating, produces numerous airborne chemicals. Secondhand smoke from tobacco and smoke from fuel combustion contain known teratogens, including polycyclic aromatic hydrocarbons (PAHs), carbon monoxide (CO), and heavy metals. Shum et al. demonstrated that periconceptional maternal exposure to the PAH benzo[a]pyrene causes cleft in genetically "nonresponsive" inbred mice, which were metabolically deficient [43]. Maternal exposure to low levels of CO has been shown to cause tissue hypoxia in rat fetuses [44], which diminishes cellular metabolism of benzo[a]pyrene [45], suggesting a potential role of $\mathrm{CO}$ in cleft development. Combustion of both tobacco and biomass fuels emit heavy metals, including cadmium, which has been shown to cause cleft development in rats $[46,47]$. At the human population level, Langlois et al. showed that maternal occupational exposure in work environments with greater levels of PAHs was associated with greater odds of cleft [42].

Bias due to control selection is a concern common to all case-control studies. Selection of more affluent controls could influence the representativeness of cooking methods in the sample with respect to the un- 
derlying base population. The replication of the association across sites (Figure 2) suggests that selection of non-comparable controls is unlikely to explain the finding due to the variability of SES and access to medical care across countries. In support of this, the association between indoor cook smoke exposure and cleft was present in countries with a variety of surgical care and those where medical missions are the primary care source. Further, differential recruitment by age of cases and controls did not explain the results as the effect magnitude was not diminished when restricting to cases one-year of age and under. The original design restricted to cases age 4 years and under to limit recall bias and in fact approximately $70 \%$ of our cases were under one year. Correction for differential selection due to SES was addressed by adjustments for household income and parental education in the analysis. Both the adjusted and stratified models by SES were consistent with the original findings.

A limitation of our study is that we cannot be certain cases are fully population-based. However, regional recruitment efforts were extensive and conducted at least four months prior to each mission. Control samples were collected from women at public neighborhood, clinic, and hospital-based birth centers to limit the oversampling of higher income families. Another concern may be underreporting of smoking or alcohol history by case mothers due to stigma around these behaviors while pregnant. While we did ask information on amount of tobacco products parents used weekly, the data was too sparse and variability too low among mothers to conduct a detailed analysis. Similarly, we did not have data on potential changes in the father's smoking habits during the pregnancy, which would not adequately classify the fathers smoking status by trimester.

\section{CONCLUSIONS}

Exposure to smoke while cooking is already a well-established health risk in low-resource countries for a wide variety of diseases but has been minimally studied with respect to cleft. We found a $50 \%$ increase in cleft risk for mothers reporting cooking over an open flame indoors compared to controls in a diverse group of LMICs. It is necessary to take risk factors specific to low resource settings into account, as those individuals are at the highest risk of being unable to access care and therefore live with the negative health consequences of disease. This information can inform public health interventions and education to potentially prevent disease in populations where care is sparse, and children are most likely to feel the detrimental, lifelong medical and social effects of cleft. Modifiable, patient-centric solutions, such as providing a clean-burning cookstove, will be critical for efforts to decrease the burden of cleft globally and improve lives around the world.

Acknowledgements: Operation Smile Global Headquarters (Norfolk, VA; Lili Arakaki); Operation Smile International (Vietnam: Thi Hai Duc Nguyen, Thuy Bui; DRC: Mwepu Idesbald Mwebe, Nunda Misabe'o Pacifique; Madagascar: Miadana Rakotondranaivo, Andriambelo Valisoa; Nicaragua: Albertina McGregor, Julio Chamorro; Philippines: Dr Irene Tangco, Dr Jello Hernandez; Honduras: Melissa Giron). University of Southern California (Annie Pham, Yvette Estrada, Louella Gonzalez, Nikki Aflatooni, Katelyn Krolick, Chantel Aftab, Ashkan Nasr, Mahmoud Hamza). Children's Hospital Los Angeles (Pedro Sanchez, Stephanie Ly), as well as all of the volunteer interviewers in each of the countries who have made this possible.

Funding: This work was supported by private funding from Operation Smile, a registered 501(c)3 not-for-profit organization. The funding organization had no role in the design or outcome of this research. The authors declare they have no actual or potential competing financial interests.

Author contributions: AA, SR, MD, FB, KM, and WM conceived the presented idea and designed the methodology. RK managed and compiled the database used for the analysis. AA performed the analysis and wrote the majority of the text. MD, FB and KM verified the methods. DC advised on and verified the analytical methods. BS edited the text and contributed to the literature review for the introduction and discussion. W.M and RM supervised the manuscript development and overall findings of this work. All authors discussed the results and contributed to the final manuscript.

Competing interests: The authors completed the ICMJE Unified Competing Interest form (available upon request from the corresponding author), and declare no conflicts of interest.

Additional material

Online Supplementary Document 
1 Meara JG, Leather AJ, Hagander L, Alkire BC, Alonso N, Ameh EA, et al. Global Surgery 2030: evidence and solutions for achieving health, welfare, and economic development. Lancet. 2015;386:569-624. Medline:25924834 doi:10.1016/ S0140-6736(15)60160-X

2 Mossey PA, Little J, Munger RG, Dixon MJ, Shaw WC. Cleft lip and palate. Lancet. 2009;374:1773-85. Medline:19747722 doi:10.1016/S0140-6736(09)60695-4

3 Gundlach KK, Maus C. Epidemiological studies on the frequency of clefts in Europe and world-wide. J Craniomaxillofac Surg. 2006;34 Suppl 2:1-2. Medline:17071381 doi:10.1016/S1010-5182(06)60001-2

4 Cooper ME, Ratay JS, Marazita ML. Asian oral-facial cleft birth prevalence. Cleft Palate Craniofac J. 2006;43:580-9. Medline:16986997 doi:10.1597/05-167

5 Kadir A, Mossey PA, Blencowe H, Moorthie S, Lawn JE, Mastroiacovo P, et al. Systematic review and meta-analysis of the birth prevalence of orofacial clefts in low- and middle-income countries. Cleft Palate Craniofac J. 2017;54:571-81. Medline:27440051 doi:10.1597/15-221

6 Calzolari E, Pierini A, Astolfi G, Bianchi F, Neville AJ, Rivieri F. Associated anomalies in multi-malformed infants with cleft lip and palate: An epidemiologic study of nearly 6 million births in 23 EUROCAT registries. Am J Med Genet A. 2007;143A:528-37. Medline:17286264 doi:10.1002/ajmg.a.31447

7 Leslie EJ, Marazita ML. Genetics of cleft lip and cleft palate. Am J Med Genet C Semin Med Genet. 2013;163C:246-58. Medline:24124047 doi:10.1002/ajmg.c.31381

8 Rittler M, Lopez-Camelo J, Castilla EE. Sex ratio and associated risk factors for 50 congenital anomaly types: clues for causal heterogeneity. Birth Defects Res A Clin Mol Teratol. 2004;70:13-9. Medline:14745890 doi:10.1002/bdra.10131

9 Liu Y, Wang B, Li Z, Zhang L, Liu J, Ren A. Indoor air pollution and the risk of orofacial clefts in a rural population in Shanxi province, China. Birth Defects Res A Clin Mol Teratol. 2016;106:708-15. Medline:27227359 doi:10.1002/ bdra. 23522

10 Mbuyi-Musanzayi S, Kayembe TJ, Kashal MK, Lukusa PT, Kalenga PM, Tshilombo FK, et al. Non-syndromic cleft lip and/or cleft palate: Epidemiology and risk factors in Lubumbashi (DR Congo), a case-control study. J Craniomaxillofac Surg. 2018;46:1051-8. Medline:29802056 doi:10.1016/j.jcms.2018.05.006

11 Sehgal M, Rizwan SA, Krishnan A. Disease burden due to biomass cooking-fuel-related household air pollution among women in India. Glob Health Action. 2014;7:25326. Medline:25373414 doi:10.3402/gha.v7.25326

12 Hackshaw A, Rodeck C, Boniface S. Maternal smoking in pregnancy and birth defects: a systematic review based on 173687 malformed cases and 11.7 million controls. Hum Reprod Update. 2011;17:589-604. Medline:21747128 doi:10.1093/humupd/dmr022

13 Wyszynski DF, Duffy DL, Beaty TH. Maternal cigarette smoking and oral clefts: a meta-analysis. Cleft Palate Craniofac J. 1997;34:206-10. Medline:9167070 doi:10.1597/1545-1569_1997_034_0206_mcsaoc_2.3.co_2

14 Xuan Z, Zhongpeng Y, Yanjun G, Jiaqi D, Yuchi Z, Bing S, et al. Maternal active smoking and risk of oral clefts: a meta-analysis. Oral Surg Oral Med Oral Pathol Oral Radiol. 2016;122:680-90. Medline:27727103 doi:10.1016/j. oooo.2016.08.007

15 Yang J, Carmichael SL, Canfield M, Song J, Shaw GM. National Birth Defects Prevention S. Socioeconomic status in relation to selected birth defects in a large multicentered US case-control study. Am J Epidemiol. 2008;167:145-54. Medline:17947220 doi:10.1093/aje/kwm283

16 Acuna-Gonzalez G, Medina-Solis CE, Maupome G, Escoffie-Ramirez M, Hernandez-Romano J, Marquez-Corona Mde L, et al. Family history and socioeconomic risk factors for non-syndromic cleft lip and palate: a matched case-control study in a less developed country. Biomedica. 2011;31:381-91. Medline:22674314

17 Jahanbin A, Shadkam E, Miri HH, Shirazi AS, Abtahi M. Maternal folic acid supplementation and the risk of oral clefts in offspring. J Craniofac Surg. 2018;29:e534-41. Medline:29762322 doi:10.1097/SCS.0000000000004488

18 Kelly D, O'Dowd T, Reulbach U. Use of folic acid supplements and risk of cleft lip and palate in infants: a populationbased cohort study. Br J Gen Pract. 2012;62:e466-72. Medline:22781994 doi:10.3399/bjgp12X652328

19 Berg E, Lie RT, Sivertsen A, Haaland OA. Parental age and the risk of isolated cleft lip: a registry-based study. Ann Epidemiol. 2015;25:942-7e1. Medline:26092647 doi:10.1016/j.annepidem.2015.05.003

20 Mai CT, Cassell CH, Meyer RE, Isenburg J, Canfield MA, Rickard R, et al. Birth defects data from population-based birth defects surveillance programs in the United States, 2007 to 2011: highlighting orofacial clefts. Birth Defects Res A Clin Mol Teratol. 2014;100:895-904. Medline:25399767 doi:10.1002/bdra.23329

21 Sivertsen A, Wilcox AJ, Skjaerven R, Vindenes HA, Abyholm F, Harville E, et al. Familial risk of oral clefts by morphological type and severity: population based cohort study of first degree relatives. BMJ. 2008;336:432-4. Medline:18250102 doi:10.1136/bmj.39458.563611.AE

22 Genisca AE, Frias JL, Broussard CS, Honein MA, Lammer EJ, Moore CA, et al. Orofacial clefts in the National Birth Defects Prevention Study, 1997-2004. Am J Med Genet A. 2009;149A:1149-58. Medline:19441124 doi:10.1002/ajmg.a.32854

23 Bell JC, Raynes-Greenow C, Turner RM, Bower C, Nassar N, O'Leary CM. Maternal alcohol consumption during pregnancy and the risk of orofacial clefts in infants: a systematic review and meta-analysis. Paediatr Perinat Epidemiol. 2014;28:322-32. Medline:24800624 doi:10.1111/ppe.12131

24 Romitti PA, Sun L, Honein MA, Reefhuis J, Correa A, Rasmussen SA. Maternal periconceptional alcohol consumption and risk of orofacial clefts. Am J Epidemiol. 2007;166:775-85. Medline:17609516 doi:10.1093/aje/kwm146

25 Figueiredo JC, Ly S, Magee KS, Ihenacho U, Baurley JW, Sanchez-Lara PA, et al. Parental risk factors for oral clefts among Central Africans, Southeast Asians, and Central Americans. Birth Defects Res A Clin Mol Teratol. 2015;103:863-79. Medline:26466527 doi:10.1002/bdra.23417 
26 Lebby KD, Tan F, Brown CP. Maternal factors and disparities associated with oral clefts. Ethn Dis. 2010;20(1 Suppl 1):S1S146. Medline:20521404

27 Figueiredo JC, Ly S, Raimondi H, Magee K, Baurley JW, Sanchez-Lara PA, et al. Genetic risk factors for orofacial clefts in Central Africans and Southeast Asians. Am J Med Genet A. 2014;164A:2572-80. Medline:25099202 doi:10.1002/ ajmg.a.36693

28 ICD-10 Classifications of Mental and Behavioural Disorder. Clinical Descriptions and Disgnostic Guidelines. 1992;Geneva. World Health Organisation.

29 The World Bank. Available: https://data.worldbank.org/indicator/SH.PRV.SMOK.FE. Accessed: 14 January 2020.

30 The Health Consequences of Smoking-50 Years of Progress: A Report of the Surgeon General. Reports of the Surgeon General. Atlanta (GA), 2014.

31 Honein MA, Rasmussen SA, Reefhuis J, Romitti PA, Lammer EJ, Sun L, et al. Maternal smoking and environmental tobacco smoke exposure and the risk of orofacial clefts. Epidemiology. 2007;18:226-33. Medline:17202867 doi:10.1097/01. ede.0000254430.61294.c0

32 Ly S, Burg ML, Ihenacho U, Brindopke F, Auslander A, Magee KS, et al. Paternal risk factors for oral clefts in Northern Africans, Southeast Asians, and Central Americans. Int J Environ Res Public Health. 2017;14:657. Medline:28629204 doi:10.3390/ijerph14060657

33 Lie RT, Wilcox AJ, Taylor J, Gjessing HK, Saugstad OD, Aabyholm F, et al. Maternal smoking and oral clefts: the role of detoxification pathway genes. Epidemiology. 2008;19:606-15. Medline:18449058 doi:10.1097/EDE.0b013e3181690731

34 Junaid M, Narayanan MBA, Jayanthi D, Kumar SGR, Selvamary AL. Association between maternal exposure to tobacco, presence of TGFA gene, and the occurrence of oral clefts. A case control study. Clin Oral Investig. 2018;22:217-23. Medline:28303469 doi:10.1007/s00784-017-2102-6

35 Jia ZL, Shi B, Chen CH, Shi JY, Wu J, Xu X. Maternal malnutrition, environmental exposure during pregnancy and the risk of non-syndromic orofacial clefts. Oral Dis. 2011;17:584-9. Medline:21535328 doi:10.1111/j.1601-0825.2011.01810.x

$36 \mathrm{Pi}$ X, Li Z, Jin L, Liu J, Zhang Y, Zhang L, et al. Secondhand smoke during the periconceptional period increases the risk for orofacial clefts in offspring. Paediatr Perinat Epidemiol. 2018;32:423-7. Medline:30048566 doi:10.1111/ppe.12497

37 Kummet CM, Moreno LM, Wilcox AJ, Romitti PA, DeRoo LA, Munger RG, et al. Passive smoke exposure as a risk factor for oral clefts-a large international population-based study. Am J Epidemiol. 2016;183:834-41. Medline:27045073 doi:10.1093/aje/kwv279

38 Hoyt AT, Canfield MA, Romitti PA, Botto LD, Anderka MT, Krikov SV, et al. Associations between maternal periconceptional exposure to secondhand tobacco smoke and major birth defects. Am J Obstet Gynecol. 2016;215:613.e1-.e11. Medline:27443814 doi:10.1016/j.ajog.2016.07.022

39 McKinney CM, Pisek A, Chowchuen B, DeRouen T, Muktabhant B, Pradubwong S, et al. Case-control study of nutritional and environmental factors and the risk of oral clefts in Thailand. Birth Defects Res A Clin Mol Teratol. 2016;106:62432. Medline:27097933 doi:10.1002/bdra.23505

40 Sabbagh HJ, Hassan MH, Innes NP, Elkodary HM, Little J, Mossey PA. Passive smoking in the etiology of non-syndromic orofacial clefts: a systematic review and meta-analysis. PLoS One. 2015;10:e0116963. Medline:25760440 doi:10.1371/ journal.pone.0116963

41 Li Z, Liu J, Ye R, Zhang L, Zheng X, Ren A. Maternal passive smoking and risk of cleft lip with or without cleft palate. Epidemiology. 2010;21:240-2. Medline:20081540 doi:10.1097/EDE.0b013e3181c9f941

42 Langlois PH, Hoyt AT, Lupo PJ, Lawson CC, Waters MA, Desrosiers TA, et al. Maternal occupational exposure to polycyclic aromatic hydrocarbons and risk of oral cleft-affected pregnancies. Cleft Palate Craniofac J. 2013;50:337-46. Medline:23136939 doi:10.1597/12-104

43 Shum S, Jensen NM, Nebert DW. The murine Ah locus: in utero toxicity and teratogenesis associated with genetic differences in benzo[a]pyrene metabolism. Teratology. 1979;20:365-76. Medline:542892 doi:10.1002/tera.1420200307

44 Garvey DJ, Longo LD. Chronic low level maternal carbon monoxide exposure and fetal growth and development. Biol Reprod. 1978;19:8-14. Medline:687711 doi:10.1095/biolreprod19.1.8

45 Schults MA, Sanen K, Godschalk RW, Theys J, van Schooten FJ, Chiu RK. Hypoxia diminishes the detoxification of the environmental mutagen benzo[a]pyrene. Mutagenesis. 2014;29:481-7. Medline:25199627 doi:10.1093/mutage/geu034

46 Chernoff N. Teratogenic effects of cadmium in rats. Teratology. 1973;8:29-32. Medline:4737490 doi:10.1002/ tera. 1420080105

47 Ferm VH. Developmental malformations induced by cadmium. A study of timed injections during embryogenesis. Biol Neonate. 1971;19:101-7. Medline:5003301 doi:10.1159/000240405 